\title{
Влияние режима получения образцов и термообработки на локальную структуру халькогенидного полупроводника $\mathrm{Ge}_{2} \mathrm{Sb}_{2} \mathrm{Te}_{5}$
}

\author{
(C) С.Н. Гарибова ${ }^{1,2}$, А.И. Исаев ${ }^{1}$, С.И. Мехтиева ${ }^{1}$, С.У. Атаева ${ }^{1}$, Р.И. Алекперов ${ }^{1,3}$ \\ ${ }^{1}$ Институт ффизики Национальной академии наук Азербайджана, \\ Az1143 Баку, Азербайджан \\ ${ }^{2}$ Университет Хазар, Департамент фризики и электроники, \\ Аz1096 Баку, Азербайджан \\ ${ }^{3}$ Азербайджанский государственный экономический университет (UNEC), \\ Az1001 Баку, Азербайджан \\ E-mail: sqaribova@rambler.ru, sqaribova1@gmail.com
}

Поступила в Редакцию 15 сентября 2021 г.

В окончательной редакции 23 октября 2021 г.

Принята к публикации 23 октября 2021 г.

\begin{abstract}
Методами рентгеновской дифракции и рамановской спектроскопии исследованы особенности фазовых переходов „аморфное состояние-кристалл“ в зависимости от способа получения образцов и термообработки, а также изменения структуры и ближнего порядка в расположении атомов халькогенидного полупроводника $\mathrm{Ge}_{20} \mathrm{Sb}_{20.5} \mathrm{Te}_{51}$. Показано, что пленки $\mathrm{Ge}_{20} \mathrm{Sb}_{20.5} \mathrm{Te}_{51}$, полученные термическим испарением на неподогреваемой подложке, являются аморфными, прошедшие термообработку при 220 и $400^{\circ} \mathrm{C}$ переходят в кристаллическую фазу с кубической и гексагональной структурой. Определены химические связи и основные структурные элементы, образующие матрицу исследованных объектов, а также изменения, происходящие в них при термообработке.
\end{abstract}

Ключевые слова: халькогенидные полупроводники, локальная структура, фазовый переход, рамановская спектроскопия.

DOI: 10.21883/FTP.2022.03.52113.9744

\section{1. Введение}

Интерес к халькогенидным стеклообразным полупроводникам (ХСП) появился после того, как в этих материалах впервые были обнаружены полупроводниковые свойства, которые сочетались со свойствами стеклообразного состояния [1]. ХСП - перспективные материалы, обладающие способностью изменять электрические, фотоэлектрические и оптические свойства под действием света, т. е. изменять показатель преломления света, край оптического поглощения. Благодаря этому находят широкое применение как активные материалы в различных электрических переключателях, запоминающих устройствах, в инфракрасной (ИК) технике и акустооптических устройствах.

Эффекты переключения и памяти в халькогенидных стеклообразных полупроводниках известны давно, но физика этих эффектов до сих пор остается не известной. В последнее время возрос интерес к разработкам элементов энергонезависимой памяти на основе фазового перехода халькогенидное стекло-кристалл, который допускает обратимое изменение фазы между стабильным аморфным и кристаллическим состояниями. Элементы памяти на основе ХСП получили название фазовой памяти с изменяемым фазовым состоянием - Phase Change Memory (PCM). Малое время записи и большой оптический контраст между аморфным и кристаллическим состояниями позволили использовать материалы $\mathrm{Ge}_{2} \mathrm{Sb}_{2} \mathrm{Te}_{5}$ для создания перезаписываемых $\mathrm{CD}$, DVD, a также Blu-ray дисков, что продемонстрировало стабильность и высокую скорость фазовых переходов в материалах этой системы [2-4].

Настоящая работа посвящена исследованию локальной структуры на уровне ближнего и среднего порядков бинарных и тройных халькогенидных материалов. В качестве объекта исследования выбран халькогенидный полупроводник $\mathrm{Ge}_{20} \mathrm{Sb}_{20.5} \mathrm{Te}_{51}$. Тройной халькогенидный полупроводник Ge-Sb-Te в зависимости от температуры и технологического режима получения может находиться в аморфных и двух кристаллических фазах - кубической и гексагональной, что должно сопровождаться изменениями параметров ближнего и среднего порядков. Методами исследования локальной структуры выбраны дифракция рентгеновских лучей и рамановская спектроскопия. Такие исследования позволяют определить параметры ближнего и среднего порядков, а также химические связи и структурные элементы матрицы образцов, находящихся как в аморфном, так и в кристаллическом состоянии.

\section{2. Изготовление образцов и методика эксперимента}

Халькогенидные полупроводники Ge-Sb-Te получены сплавлением элементарных веществ особой чистоты в вакуумированных до $10^{-4}$ мм рт.ст. кварцевых ампулах. Синтез проводили при температуре $900^{\circ} \mathrm{C}$ в течение 
10 ч, с выдержкой по меньшей мере 5 ч во вращающемся термостате с последующим охлаждением в режиме выключенного термостата. В качестве образцов для измерений были использованы пленки толщиной 0.5-1.5 и 3-8мкм, полученные термическим испарением со скоростью $0.2-0.5$ мкм/с в вакууме на стеклянную подложку при давлении $10^{-4}$ мм рт.ст., а также объемные образцы толщиной 1.5 мм. Рентгеноструктурный анализ проводили с использованием дифрактометра D2Phaser фирмы „Bruker“ и $\mathrm{Cu} K_{\alpha}$-лучей с длиной волны излучения $\lambda=1.5406 \AA$ в диапазоне углов $2 \theta=5-80^{\circ}$. Структурные исследования и расчеты проводились с помощью программ EVA и TOPAZ. Рамановские спектры исследовались на трехмерном конфокальном рамановском микроскопе Nanofinder 30 (Tokyo Instr.), длина волны возбуждения составляла 532 нм. Радиус сечения падающего на пленку лазерного луча был $\sim 4$ мкм. Приемником излучения служила охлаждаемая CCD-камера $\left(-70^{\circ} \mathrm{C}\right)$, работающая в режиме счета фотонов, время экспозиции было 10-40 с при мощности лазерного излучения 9 мВт, погрешность спектрального разрешения $0.5 \mathrm{~cm}^{-1}$. Эксперименты были проведены при комнатной температуре и после термообработки образцов при 120 , $220,400^{\circ} \mathrm{C}$ в течение 30 мин.

\section{3. Экспериментальные результаты и обсуждение}

На рис. 1 представлены угловые распределения интенсивности рентгеновской дифракции образцов системы $\mathrm{Ge}_{20} \mathrm{Sb}_{20.5} \mathrm{Te}_{51}$. Как видно из рентгенограммы, пленочный образец при комнатной температуре (рис. 1, $a$, кривая 1) имеет широкий максимум при значениях $2 \theta$ $\left(\theta-\right.$ угол Брэгга), расположенных в интервале $15-38^{\circ}$, что свидетельствует об их аморфности. Аналогичной протяженностью обладает большинство ХСП [5,6]. Рентгеновский спектр объемного образца Ge-Sb-Te толщиной $1.5 \mathrm{Mм}$ (рис. 1,a, кривая 2) показывает, что до температуры обработки наличие пиков при углах 25.7, 29.7, 42.8 и 52.9 ${ }^{\circ}$ указывает на кубическую фазу кристаллической структуры.

На рис. $1, b$ показаны рентгеновские спектры образцов $\mathrm{Ge}_{20} \mathrm{Sb}_{20.5} \mathrm{Te}_{51}$ до (кривая 1) и после термообработки (кривые 2-4). Дифракционная картина объемного образца до термообработки (рис. $1, b$, кривая 1 ), полученная в режиме выключенной печи, обладает максимумами (reflections) при углах $25.7(111), 29.7$ (200), 42.8 (220) и $52.9^{\circ}(222)$, соответствующих отражениям первого и второго порядков в метастабильной гранецентрированной кубической (face-centered cubic - FCC) фазе вдоль (111) направления [7,8]. При температуре $120^{\circ} \mathrm{C}$ пики в пленочном образце (рис. $1, b$, кривая 2) остаются почти неизменными, но в пленках (рис. 1, $b$, кривые 3,4) при тех же значениях угла появляются пики, свидетельствующие о кристаллизации. При температуре $220^{\circ} \mathrm{C}$ интенсивности указанных пиков увеличиваются и
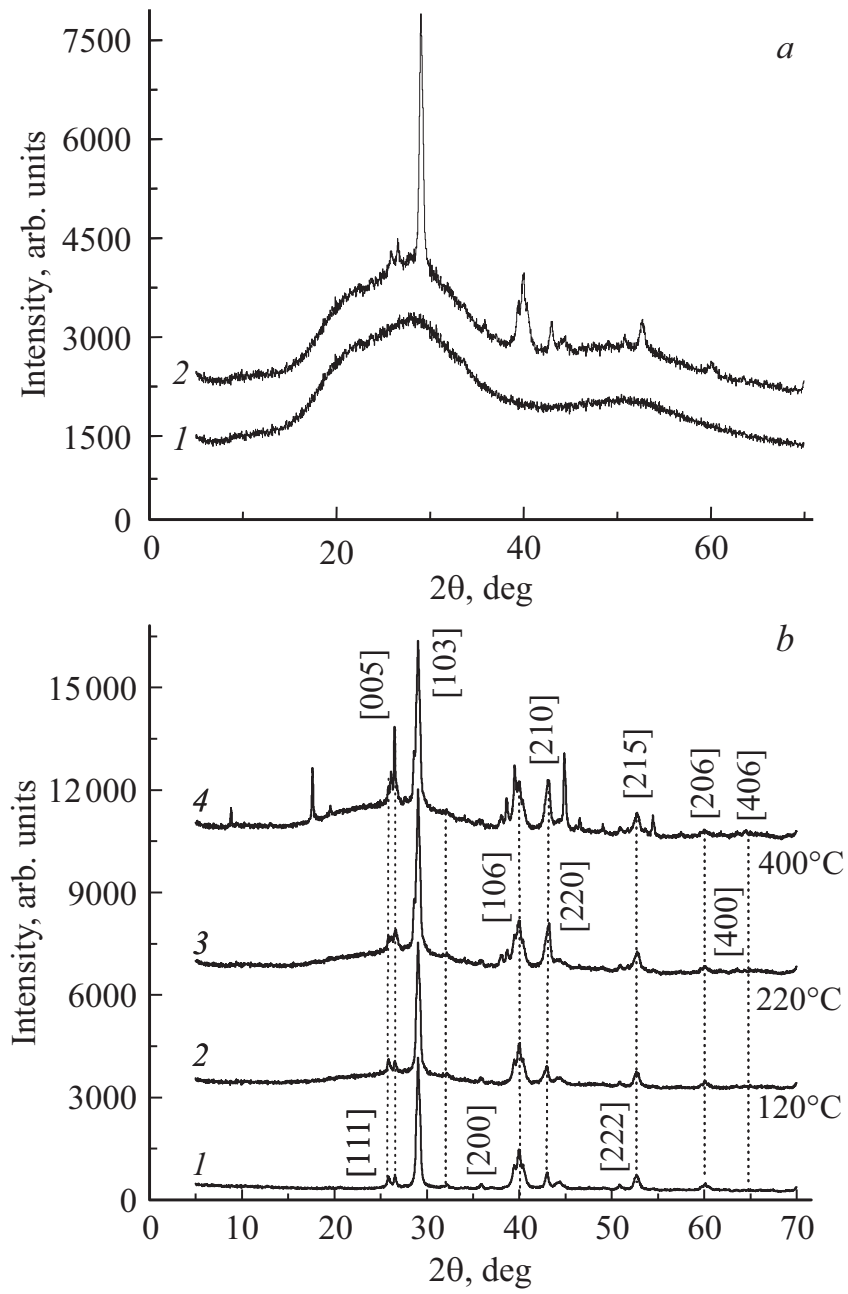

Рис. 1. Угловые распределения интенсивности рентгеновской дифракции $\mathrm{Ge}_{20} \mathrm{Sb}_{20.5} \mathrm{Te}_{51}: a-$ при комнатной температуре (кривая 1 - пленочный образец, 2 - объемный); $b$ - до (кривая 1) и после термообработки (кривые 2-4).

появляются новые при 29.7 (103), 40.1 (106), 42.8 (210), $60.1(206), 63.60^{\circ}(400)$, интенсивности которых также увеличиваются с дальнейшим ростом термообработки (до $400^{\circ} \mathrm{C}$ ). Положения фиксируемых пиков соответствуют результатам работ [7-9] и свидетельствуют о происхождении структурных изменений образца на стабильной гексагональной фазе (HEX - phase structure).

Материалы системы Ge-Sb-Te обладают низкой стеклообразующей способностью и легко кристаллизуются, и, чтобы получить такой материал в аморфном состоянии, нужно охладить расплав за несколько десятков или сотен наносекунд. Именно высокая скорость кристаллизации позволяет в современных ячейках памяти записывать информацию за время < 50 нс. Однако при понижении температуры время кристаллизации экспоненциально растет и при комнатной температуре составляет около 100 лет [10]. Поэтому материалы системы $\mathrm{Ge}-\mathrm{Sb}$-Те успешно используются в современных носителях информации. 

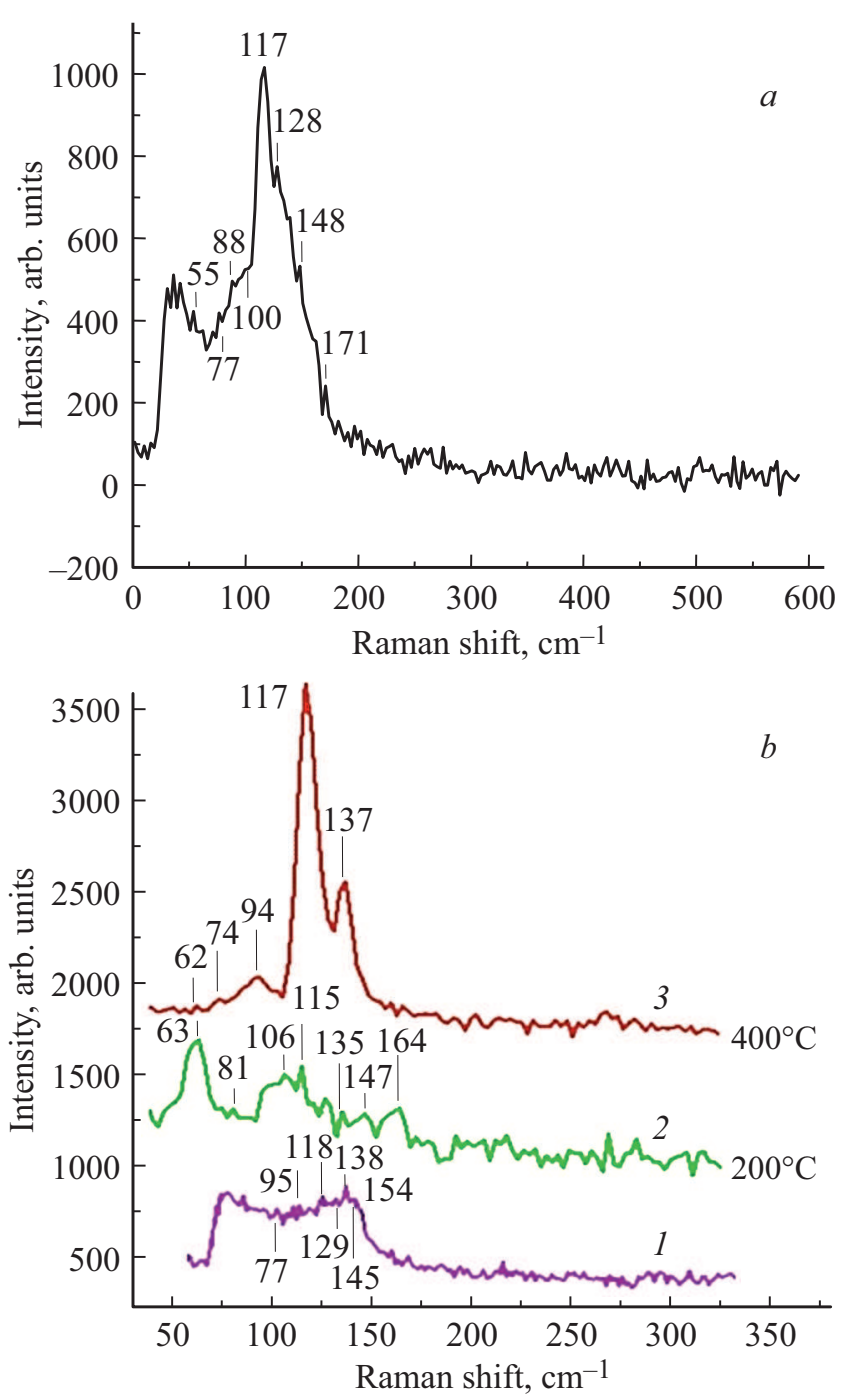

Рис. 2. Рамановские спектры: $a$ - объемного образца $\mathrm{Ge}-\mathrm{Sb}-\mathrm{Te}$ до термообработки; $b-$ пленочных образцов $\mathrm{Ge}-\mathrm{Sb}$-Те после термообработки.

Анализ рентгенограмм $\mathrm{Ge}_{20} \mathrm{Sb}_{20.5} \mathrm{Te}_{51}$ указывает на то, что пленочные образцы до термообработки являются аморфными, в то время как объемный образец имеет кристаллическую структуру, в частности кубическую. После температуры обработки (при $120^{\circ} \mathrm{C}$ ) пленочные образцы кристаллизуются, частично переходя в кубическую структуру. С дальнейшим ростом температуры до $220^{\circ} \mathrm{C}$ в структуре пленок появляются пики, указывающие на гексагональную фазу, при $400^{\circ} \mathrm{C}-$ переходит в стабильную.

На рис. 2 показаны рамановские спектры объемного (рис. 2,a) и пленочного (рис. 2, $b$ ) образцов $\mathrm{Ge}_{20} \mathrm{Sb}_{20.5} \mathrm{Te}_{51}$, полученные при комнатной температуре (кривая 1) и подвергавшиеся термической обработке при температурах 220 (кривая 2) и $400^{\circ} \mathrm{C}$ (кривая 3). Спектр пленок при комнатной температуре состоит из широкой полосы, охватывающей интервал частот $75-175 \mathrm{~cm}^{-1}$ со слабыми максимумами при 95, 105,
$118,129,147$ и $160 \mathrm{~cm}^{-1}$ (рис. 2, $b$, кривая 2). Максимумы при $160,149,129 \mathrm{~cm}^{-1}$, с некоторыми отклонениями наблюдались в рамановском спектре объемных образцов (рис. 2,a) и пленок, полученных до и после термообработки при $220^{\circ} \mathrm{C}$ (рис. $2, b$ кривые 1 и 2), а максимумы при $115-117$ и $74-90 \mathrm{~cm}^{-1}-$ в спектре всех образцов. Работы [11-18] также посвящены исследованию особенностей рамановских спектров аморфных и кристаллических образцов $\mathrm{Ge}_{2} \mathrm{Sb}_{2} \mathrm{Te}_{5}$. Авторы $[11,15]$ приписывали пик $160 \mathrm{~cm}^{-1}$ к колебаниям дефектных октаэдрических структурных элементов на основе Ge. B работе [15] методами из первых принципов ( $a b$ initio) и эмпирической моделью поляризуемости связей были рассчитаны рамановские спектры аморфной и кристаллы кубической структуры $\mathrm{Ge}_{2} \mathrm{Sb}_{2} \mathrm{Te}_{5}$. Авторы пришли к выводу, что наблюдаемые особенности как аморфного, так и кристаллического $\mathrm{Ge}_{2} \mathrm{Sb}_{2} \mathrm{Te}_{5}$ можно отнести к колебаниям дефектных октаэдров. Признаки тетраэдров в спектре рамановского рассеяния аморфных образцов покрыты большим поперечным сечением рамановского рассеяния дефектных октаэдров. Авторами работы [12] указанный пик был приписан колебаниям гомеополярной связи $\mathrm{Sb}-\mathrm{Sb}$, a авторами [13] - к кристаллизации образцов. Авторами работ $[15,17,18]$ максимум при $160 \mathrm{~cm}^{-1}$ связывался с активацией колебательных мод $\mathrm{A}_{1 g}$, и их появление считалось признаком образования кристаллических структурных элементов.

Полоса, локализованная на частоте $\sim 150 \mathrm{~cm}^{-1}$ (в наших исследованиях при $\sim 147 \mathrm{~cm}^{-1}$ ), непосредственно связана со структурным элементом $\mathrm{Sb}_{2} \mathrm{Te}_{3}$ и частично связана с пирамидальным структурным элементом $\mathrm{SbTe}_{3}$ [11]. В исследованиях полоса с максимумами $145-150 \mathrm{~cm}^{-1}$ связывалась с колебаниями связи $\mathrm{Sb}-\mathrm{Te}$ в пирамидальном структурном элементе $\mathrm{SbTe}_{3}$ [18] или же в октаэдрически координированных атомах $\mathrm{Sb}$ [15]. Широкая полоса при $150 \mathrm{~cm}^{-1}$ также наблюдалась в работе [19] и связывалась с колебаниями гомеополярной связи Те-Те. Авторы указанной работы отметили, что данная полоса наблюдается только в образцах, подвергшихся низкотемпературному отжигу. Пик при $150 \mathrm{~cm}^{-1}$, соответствующий модам растяжения связи $\mathrm{Te}-\mathrm{Te}$, не проявлялся в рамановском спектре объемных образцов $\mathrm{Ge}_{2} \mathrm{Sb}_{2} \mathrm{Te}_{5}$ [13], что считалось признаком хорошей кристаллизации.

Пики при частотах $\sim 127-129 \mathrm{~cm}^{-1}$ в рамановском спектре наблюдались во всех образцах $\mathrm{Ge}_{2} \mathrm{Sb}_{2} \mathrm{Te}_{5}$, кроме пленочных, подвергшихся термообработке при $400^{\circ} \mathrm{C}$. Положение этого пика очень близко максимуму при $125 \mathrm{~cm}^{-1}$, наблюдаемому в работе [11], которое авторами статьи [20] с учетом результатов исследований рамановского рассеяния в тонких пленках $a$-GeTe [21] было приписано моде $A_{1} \mathrm{GeTe}_{4-n} \mathrm{Ge}_{n}(n=1,2)$ (corner-sharing tetrahedral). О существовании тетраэдрического звена $\mathrm{GeTe}_{4-n} \mathrm{Ge}_{n} \quad(n=1,2)$, включая гомеополярные связи $\mathrm{Ge}-\mathrm{Ge}$, также сообщено в работе [22], как результате исследования EXAFS. Тетраэдрические структурные 

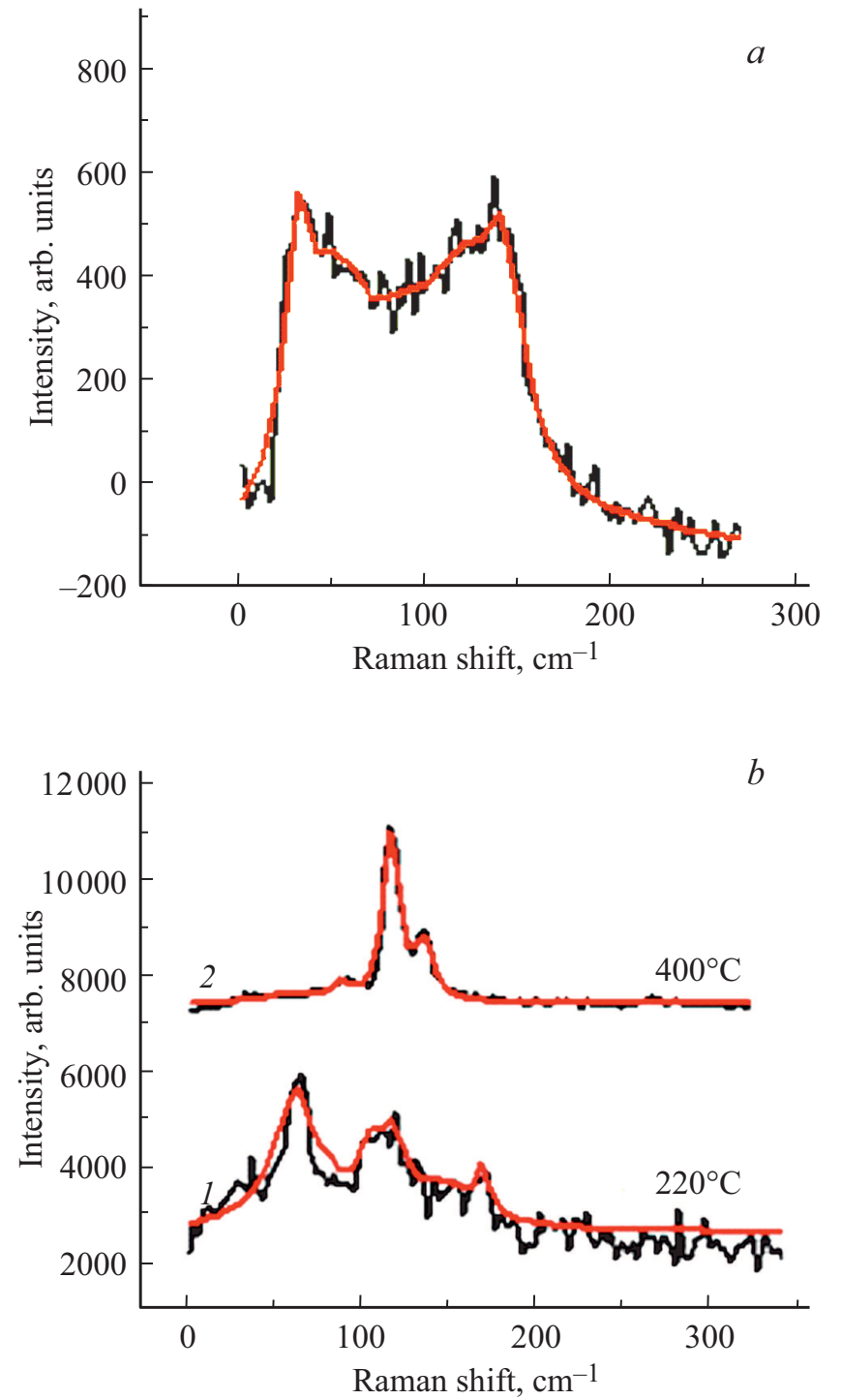

Data: Data1 B

Model: Lorentz

Equation: $\mathrm{y}=\mathrm{y} 0+(2 \cdot \mathrm{A} / \mathrm{PI}) \cdot\left(\mathrm{w} /\left(4 \cdot(\mathrm{x}-\mathrm{xc})^{2}+\mathrm{w}^{2}\right)\right)$

Weighting:

y No weighfing

$\mathrm{Chi}^{2} / \mathrm{DoF}=1542.38341$

$\mathrm{R}^{2}=0.97466$

y0 $12.0989 \quad \pm 11.99347$

$\begin{array}{lll}\mathrm{xc} 1 & 143.34636 & \pm 1.55681\end{array}$

w1 $23.67131 \quad \pm 10.37271$

A1 $12633.16408 \pm 12019.90827$

$\begin{array}{lll}\mathrm{xc} 2 & 32.70279 & \pm 0.74018\end{array}$

$\begin{array}{lll}\text { w2 } & 18.11962 \quad \pm 4.3182\end{array}$

A2 $13633.74564 \pm 5654.14565$

$\begin{array}{lll}\mathrm{xc} 3 & 54.72571 \quad \pm 3.22804\end{array}$

$\begin{array}{lll}\text { w3 } & 39.8164 & \pm 17.89813\end{array}$

A3 $24431.68642 \pm 19608.93905$

$\begin{array}{lll}\mathrm{xc} 4 & 216.64258 \quad \pm 110.00781\end{array}$

w4 $217.85889 \quad \pm 196.59673$

A4 $16144.31206 \quad \pm 29529.24145$

$\begin{array}{lll}\mathrm{xc5} & 86.7971 \quad \pm 6.76669\end{array}$

w5 $44.39185 \quad \pm 47.87346$

A5 $\quad 14648.06188 \quad \pm 31113.88981$

$\begin{array}{lll}\text { xc6 } & 120.75529 & \pm 7.62282\end{array}$

$\begin{array}{lll}\text { w6 } & 55.59526 & \pm 33.62777\end{array}$

A6 $37567.41788 \quad \pm 43461.29964$

Data: Data1 B

Model: Lorentz

Equation: $\mathrm{y}=\mathrm{y} 0+(2 \cdot \mathrm{A} / \mathrm{PI}) \cdot\left(\mathrm{w} /\left(4 \cdot(\mathrm{x}-\mathrm{xc})^{2}+\mathrm{w}^{2}\right)\right)$

Weighting:

y No weighfing

$\mathrm{Chi}^{2} / \mathrm{DoF}=32758.77295$

$\mathrm{R}^{2}=0.98204$

y0 $\quad 3030.40565 \quad \pm 24.82442$

Xc1 $117.76883 \quad \pm 0.10917$

w1 $10.26252 \quad \pm 0.37422$

A1 $121818.02487 \pm 3629.25142$

$\begin{array}{lll}\mathrm{xc} 2 & 136.44718 \quad \pm 0.33242\end{array}$

w2 $10.97509 \quad \pm 1.07932$

A2 $44513.18654 \pm 3583.77766$

$\begin{array}{lll}\mathrm{xc} 3 & 89.34264 \quad \pm 1.27677\end{array}$

w3 $12.05023 \quad \pm 4.56792$

A3 $13163.99109 \pm 4451.75704$

xc4 $55.63243 \quad \pm 5.33923$

w4 $\quad 37.0987 \quad \pm 18.27381$

A4 $19557.72838 \pm 8379.19675$

Рис. 3. Распределение Лоренца для пиков пленочных образцов $\mathrm{Ge}_{2} \mathrm{Sb}_{2} \mathrm{Te}_{5}(a)$ аморфного и обработанных $(b)$ при температурах 220 и $400^{\circ} \mathrm{C}$.

элементы также присущи бинарным халькогенидам типа $\mathrm{GeSe}_{2}$ и $\mathrm{GeS}_{2}[23,24]$.

Как видно из рис. 2, пик при $115-118 \mathrm{~cm}^{-1}$, очень слабо наблюдаемый в рамановском спектре пленок (рис. 2,b), полученных при комнатной температуре (кривая 1), становится ярко выраженным после термообработки при $220^{\circ} \mathrm{C}$ (кривая 2) и сильно усиливается при $400^{\circ} \mathrm{C}$ (кривая 3). Указанный пик также достаточно интенсивно будет наблюдаться в объемных образцах (рис. 2,a). Пик при $\sim 115 \mathrm{~cm}^{-1}$ также наблюдается в работе [13], а пик при $120 \mathrm{~cm}^{-1}$ - в работах [14,15,17,25], которые приписаны к колебанию дефектных октаэдров. Слабые пики в интервале частот 74-90 $\mathrm{cm}^{-1}$, наблюдаемые в рамановском спектре всех образцов, также были зарегистрированы в работах [20,26-28], и авторы работы [20], учитывая результаты исследований, представленные в статье [26], связывали с $E$ модой тетраэдры $\mathrm{GeTe}_{4}$.

На рис. 3 показана точная идентификация пиков с помощью распределения Лоренца для пленочных образцов $\mathrm{Ge}_{2} \mathrm{Sb}_{2} \mathrm{Te}_{5}$ до и после термообработки. Как видно из численных значений, при переходе из аморфного состояния (рис. $3, a)$ в кубическую структуру (рис. $3, b$, кривая 1 ), а затем в гексагональную (рис. $3, b$, кривая 2) расположение атомов в указанных фазах по мере возрастания температуры термообработки пленок - разное. Ширина пиков сужается, что свидетельствует о фазовом переходе „аморфное состояние-кристалл“. 
В образцах, прошедших термообработку при $220^{\circ} \mathrm{C}$, интенсивность пиков при $\sim 127-129 \mathrm{~cm}^{-1}$ и $\sim 145-150 \mathrm{~cm}^{-1}$ уменьшается и появляются пики при $\sim 105-106 \mathrm{~cm}^{-1}$ и $\sim 135-137 \mathrm{~cm}^{-1}$ колебательных мод для кристаллических фаз кубической структуры (рис. $1, b)$. Согласно работе [8], первый пик можно отнести к колебаниям $A_{1}$ тетраэдров $\mathrm{GeTe}_{4}$, а второй к $A_{1}$ тетраэдров $\mathrm{GeTe}_{4-n} \mathrm{Ge}_{n}(n=1,2)$. В образцах, прошедших термообработку при $400^{\circ} \mathrm{C}$, указанные пики исчезают и остаются низкочастотные, появляются ярко выраженные при 117 и $137 \mathrm{~cm}^{-1}$, т.е. образуются кристаллические фазы с гексагональной структурой (рис. $1, b$ ).

Из приведенной интерпретации результатов по исследованию методом рамановской спектроскопии образцов $\mathrm{Ge}_{20} \mathrm{Sb}_{20.5} \mathrm{Te}_{51}$, полученных различными способами (термическим напылением и охлаждением сплава), а также кристаллизованных при термообработке, видно, что положения некоторых пиков совпадают, что свидетельствует о совпадении ближнего порядка в расположении атомов.

\section{4. Заключение}

Установлено, что пленочные образцы $\mathrm{Ge}_{20} \mathrm{Sb}_{20.5} \mathrm{Te}_{5}$, полученные термическим напылением на неподогреваемой подложке, являются аморфными, прошедшие термообработку при температурах 220 и $400^{\circ} \mathrm{C}$ переходят в кристаллическую фазу с кубической (FCC) и гексагональной (HEX) структурой, а образцы, полученные охлаждением расплава, частично кристаллизуются.

Анализ рамановских спектров с учетом литературных данных, а также выполненная идентификация пиков с помощью распределения Лоренца позволили определить химические связи и основные структурные элементы, образующие матрицу исследованных объектов, а также изменения, происходящие в них при термообработке. Показано, что основными структурными элементами объемных образцов и пленок, полученных термическим напылением на неподогреваемой подложке и обработанных при температуре $220^{\circ} \mathrm{C}$, являются дефектные октаэдры $\mathrm{GeTe}_{4-n} \mathrm{Ge}_{n}(n=1,2)$, структурный элемент $\mathrm{Sb}_{2} \mathrm{Te}_{3}$ и пирамидальный структурный элемент $\mathrm{SbTe}_{3}$.

\section{Финансирование работы}

Работа выполнена в Институте физики Национальной академии наук Азербайджана.

\section{Конфликт интересов}

Авторы заявляют, что у них нет конфликта интересов.

\section{Список литературы}

[1] B.T. Kolomies, N.A. Qoryunova. Techn. Phys. Lett., 25 (6), 984 (1955) [in Russian].

[2] M. Wuttig, N. Yamada. Nature Materials, 6, 824 (2007).
[3] W. Welnic, M. Wuttig. Materials Today, 11 (6), 20 (2008).

[4] D. Lencer, M. Salinga, M. Wuttig. Adv. Mater., 23, 2030 (2011).

[5] S.N. Garibova, A.I. Isaev, S.I. Mekhtieva, S.U. Ataeva. Semiconductors, 53 (11), 1507 (2019).

[6] S.U. Ataeva, S.I. Mekhtieva, A.I. Isaev, S.N. Garibova, A.S. Huseynova. Semiconductors, 53, 1637 (2019).

[7] V. Bragaglia, B. Jenichen, A. Giussani, K. Perumal, H. Riechert, R. Calarco. Appl. Phys., 116, 054913 (2014).

[8] X. Zemin, C. Chaonan, W. Zhewei, W. Ke, C. Haining, Y. Hui. RSC Advances, 8, 21040 (2018).

[9] Zhang Ting, Liu Bo, Xia Ji-Lin, Song Zhi-Tang, Feng SongLin, Chen Bomy. Chin. Phys. Lett., 21 (4), 741 (2004).

[10] A.L. Lacaita. Sol. St. Electron., 50, 24 (2006).

[11] K. Shportko, 1. Revutska, O. Paiuk, J. Baran, A. Stronski, A. Gubanova, E. Venger. Optical Mater., (Amst), 73, 489 (2015).

[12] B. Liu, Z. Song, T. Zhang, S. Feng, B. Chen. Chinese Phys., 13, 1947 (2004).

[13] E. Cho, S. Yoon, H.R. Yoon, W. Jo. J. Korean Phys. Soc., 48 (6), 1616 (2006).

[14] V. Bragaglia, K. Holldack, J.E. Boschker, F. Arciprete, E. Zallo, T. Flissikowski, R. Calarco. Sci. Rept., 6, 28560 (2016).

[15] G.G. Sosso, S. Caravati, R. Mazzarello, M. Bernasconi. Phys. Rev. B, 83, 134201 (2011).

[16] S.A. Kozyukhin, V.H. Kudoyarova, H.P. Nguyen, A. Smirnov, V. Lebedev. Phys. Status Solidi C, 8 (9), 2688 (2011).

[17] G. Bulai, O. Pompilian, S. Gurlui, P. Nemec, V. Nazabal, N. Cimpoesu, B. Chazallon, C. Focsa. Nanomaterials, 9, 676 (2019).

[18] P. Nemec, V. Nazabal, A. Moreac, J. Gutwirth, L. Benes, M. Frumar. Mater. Chem. Phys., 136, 935 (2012).

[19] J. Tominaga, N. Atoda. Jpn. J. Appl. Phys., 38, L322 (1999).

[20] S. Kozyukhin, M. Veres, H.P. Nguyen, A. Ingram, V. Kudoyarova. Phys. Procedia, 44, 82 (2013).

[21] K.S. Andrikopoulos, S.N. Yannopoulos, A.V. Kolobov, P. Fons, J. Tominaga. J. Phys.: Condens. Matter, 18 (2006).

[22] G. Lucovsky, D.A. Baker, M.A. Paesler, J.C. Phillips. J. NonCryst. Sol., 353, 1713 (2007).

[23] S. Sugai. Phys. Rev. B, 35, 1345 (1987).

[24] J. Koblar, B. Arlin, G. Shau. Phys. Rev. B, 60, R14985(R) (1999).

[25] E. Yalon, S. Deshmukh, M. Munoz Rojo, F. Lian, C.M. Neumann, F. Xiong, E. Pop. Sci. Rept., 7, 15360 (2017).

[26] K.S. Andrikopoulos, S.N. Yannopoulos, A.V. Kolobov, P. Fons, J. Tominaga. J. Phys. Condens. Matter, 18 (2006).

[27] P. Nemec, A. Moreac, V. Nazabal, M. Pavlišta, J. Prikryl, M. Frumar. J. Appl. Phys., 106, 103509 (2009).

[28] H.R. Yoo, W. Jo, E. Cho, S. Yoon, M. Kim. J. Non-Cryst. Sol., 352, 3757 (2006).

Редактор А.Н. Смирнов 


\section{Influence of samples obtaining mode and heat treatment on local structure of chalcogenide semiconductor $\mathrm{Ge}_{2} \mathrm{Sb}_{2} \mathrm{Te}_{5}$}

S.N. Garibova ${ }^{1,2}$, A.I. Isaev ${ }^{1}$, S.I. Mekhtieva ${ }^{1}$, S.U. Ataeva ${ }^{1}$, R.I. Alekberov ${ }^{1,3}$

${ }^{1}$ Institute of Physics

of Azerbaijan National Academy of Sciences, Az1143 Baku, Azerbaijan

${ }^{2}$ Khazar University, Department of Physics and Electronics, Az1143 Baku, Azerbaijan

${ }^{3}$ Azerbaijan State University of Economics (UNEC), Az1001 Baku, Azerbaijan

Abstract Specifics of ,amorphous state-crystal“ phase transitions in dependence on the samples obtaining method and thermal processing, as well as changes in the structure and close range order in the arrangement of the atoms of $\mathrm{Ge}_{20} \mathrm{Sb}_{20.5} \mathrm{Te}_{51}$ chalcogenide semiconductors have been studied by the $X$-ray diffraction and Raman spectroscopy. It has been shown that $\mathrm{Ge}_{20} \mathrm{Sb}_{20.5} \mathrm{Te}_{51}$ films obtained by thermal evaporation on an unheated substrate are amorphous; after heat treatment at 220 and $400^{\circ} \mathrm{C}$, transform into a crystalline phase with a cubic and hexagonal structure. The chemical bonds and the main structural elements that form the matrix of the investigated objects, as well as the changes that occur in them during heat treatment, have been determined. 